\title{
STATIC RESONANCE IN ROTATING NANOBARS
}

\author{
UĞUR GüVEN \\ Yildiz Technical University, Department of Mechanical Engineering, Istanbul, Turkey \\ e-mail:uguven@yildiz.edu.tr
}

\begin{abstract}
In this study, static resonance that occurs in rotating nanobars is addressed. The analysis is based on Eringen's nonlocal elasticity theory and is performed in Lagrangian coordinates. Explicit solutions are given for both clamped-free and clamped-clamped boundary conditions. The present study shows that the static resonance phenomenon is largely a critical case requiring attention for rotating nanobars with small lengths.
\end{abstract}

Keywords: rotating nanobar, static resonance, nonlocal elasticity, lagrangian coordinates

\section{Introduction}

The rotating bars have attracted considerable attention in mechanical and aerospace engineering applications as machine elements such as turbines, propellers and helicopter blades. As is known, when the angular velocity of the bar reaches a certain critical value, the static resonance occurs and the longitudinal displacement becomes unbounded. This phenomenon has been first noticed by Bhuta and Jones (1963) and it has been extended by Brunelle (1971) for the rotating disks. As pointed out in (1963), the use of Eulerian coordinates does not even show this resonant character. In those analyses (Bhuta and Jones, 1963; Brunelle, 1971) Lagrangian coordinates were used. Shum and Entwistle (2006) reported that the linear uniaxial model is not representative for the situation at larger strains due to higher angular velocity. The axial deformation of rotating rods was investigated (Hodges and Bless, 1994) by using two simpler nonlinear strain energy models.

Nowadays, the recent developments in science and technology has enabled production of various rotating structures in micro and nano scales. Some publications in this new field can be found (Narendar, 2011, 2012; Narendar and Gopalakrishnan, 2011; Aranda et al., 2012; Danesh and Asghari, 2014) in literature. However, no analytical or numerical study of the static resonance in the nanobars has yet been done. The aim of this work is to investigate the scale effect on the static resonance. In this analysis, the equation of motion is formulated in the Lagrangian coordinates and Eringen's nonlocal elasticity theory is adopted. In this study, the static resonance phenomenon in rotating nanobars is addressed for two boundary conditions: clamped-free (C-F) and clamped-clamped (C-C). It can be seen from the present analysis that the results presented are strongly affected with the boundary conditions (C-F or C-C) and the coordinates systems (Eulerian or Lagrangian).

\section{Formulation of the problem and nonlocal elasticity solution}

A uniform nanobar of length $L$ rotating statically about the axis of rotation with angular velocity $\Omega$ is shown in Fig. 1 .

The equation of motion in the Lagrangian coordinates is expressed (Bhuta and Jones, 1963) as

$$
\frac{\partial \sigma}{\partial x}+\rho \Omega^{2}(u+x)=0
$$




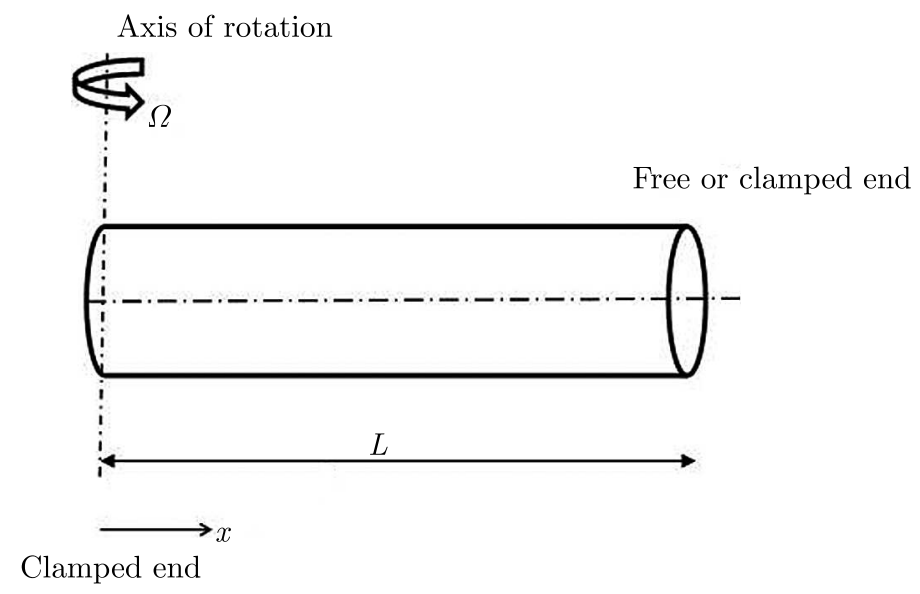

Fig. 1. Sketch of the rotating nanobar

where $\sigma$ is the nonlocal longitudinal stress, $\rho$ is density, $x$ is the axial distance and $u$ is the longitudinal displacement.

Eringen's nonlocal elasticity theory (Eringen, 2002) for one dimensional case can be expressed in the following form

$$
\sigma-\left(e_{0} a\right)^{2} \frac{\partial^{2} \sigma}{\partial x^{2}}=\sigma^{l o c a l}=E \frac{\partial u}{\partial x}
$$

where $e_{o} a$ is the small scale coefficient.

By using Eqs. (2.1) and (2.2), the nonlocal longitudinal stress is obtained as follows

$$
\sigma=E \frac{\partial u}{\partial x}-\left(e_{0} a\right)^{2} \rho \Omega^{2}\left(1+\frac{\partial u}{\partial x}\right)
$$

Substituting Eq. (2.3) into Eq. (2.1), the governing equation is given by

$$
E \frac{\partial^{2} u}{\partial x^{2}}-\left(e_{0} a\right)^{2} \rho \Omega^{2} \frac{\partial^{2} u}{\partial x^{2}}+\rho \Omega^{2} u=-\rho \Omega^{2} x
$$

and the general solution to Eq. (2.4) becomes as

$$
u=C_{1} \sin k x+C_{2} \cos k x-x
$$

where $C_{1}$ and $C_{2}$ are integration constants and $k^{2}=\rho \Omega^{2} /\left[E-\left(e_{0} a\right)^{2} \rho \Omega^{2}\right]$.

For the clamped-free boundary conditions, i.e. $u(0)=0$ and $u^{\prime}(L)=0$, the longitudinal displacement $u$ is given by

$$
u=\frac{\sin k x}{k \cos k L}\left[1+\frac{\left(e_{0} a\right)^{2} \rho \Omega^{2}}{E-\left(e_{0} a\right)^{2} \rho \Omega^{2}}\right]-x
$$

provided that $\Omega$ does not correspond to a root of

$$
\cos k L=0
$$

When $\Omega$ corresponds to a root of Eq. (2.7)

$$
\Omega_{n}=\sqrt{\frac{\frac{E}{\rho}\left[(2 n-1) \frac{\pi}{2 L}\right]^{2}}{1+\left(e_{0} a\right)^{2}\left[(2 n-1) \frac{\pi}{2 L}\right]^{2}}}
$$

static resonances occur. The practical value of the critical angular velocity is obtained for $n=1$. 
On the other hand, in the Eulerian coordinates, i.e. neglecting the longitudinal displacement term $u$ in Eq. (2.1), and by repeating the previous similar operations, the final form of the longitudinal displacement is obtained as

$$
u=\frac{\rho \Omega^{2}}{2 E}\left\{\left[L^{2}+2\left(e_{0} a\right)^{2}\right] x-\frac{x^{2}}{3}\right\}
$$

Thus, Eq. (2.9) shows clearly that the static resonance phenomenon would not be noticed (i.e., the longitudinal displacement can not become unbounded for a certain value of the angular velocity), for clamped-free boundary conditions when the Eulerian coordinates are used.

Secondly, for the clamped-clamped boundary conditions, i.e. $u(0)=0$ and $u(L)=0$, the longitudinal displacement $u$ is given by

$$
u=\frac{L \sin k x}{\sin k L}-x
$$

provided that $\Omega$ does not correspond to a root of

$$
\sin k L=0
$$

When $\Omega$ corresponds to a root of Eq. (2.11)

$$
\Omega_{n}=\sqrt{\frac{\frac{E}{\rho}\left(\frac{n \pi}{L}\right)^{2}}{1+\left(e_{0} a^{2} \frac{n \pi}{L}\right)^{2}}}
$$

static resonances occurs. The critical angular velocity of practical interest is obtained for $n=1$.

If the Eulerian coordinates are used in the same analysis, longitudinal displacement expression (2.10) takes the following form

$$
u=\frac{\rho \Omega^{2}}{6 E}\left(L^{2}-x^{2}\right) x
$$

Thus, Eq. (2.13) shows clearly that the static resonance phenomenon of motion can not be seen for the clamped-clamped boundary conditions if the Eulerian coordinates are used and, furthermore, the longitudinal displacement is independent of the effect of the small scale coefficient.

\section{Numerical example}

In this Section, for a numerical example as in (Narendar and Gopalakrishnan, 2011), $a(5,5)$ SWCNT is considered. The diameter is $d=0.675 \mathrm{~nm}$, length $L=10 d$, the elasticity modulus $E=5.5 \mathrm{TPa}$ and density $2300 \mathrm{~kg} / \mathrm{m}^{3}$. In the numerical illustration the following defined ratio is used:

Critical angular velocities ratio $=$ Critical angular velocity calculated from the nonlocal elasticity theory/Critical angular velocity calculated from the classical elasticity theory

Figure 2 shows the critical angular velocities ratio with the dimensionless scale coefficient $e_{0} a / L$, for the clamped-free and the clamped-clamped boundary conditions. From Fig. 2 it is found that as the scale coefficient $e_{0} a$ increases, the critical angular velocity decreases. The classical elasticity solution overestimates the critical angular velocities compared to the nonlocal elasticity solution. In addition, for the clamped-free boundary condition, the critical angular velocities are found to be higher compared to those for the clamped-clamped boundary condition. For the range of small scale parameters in Fig. 2, a detailed previous reference work (Narendar et al., 2011) has been taken into consideration. 


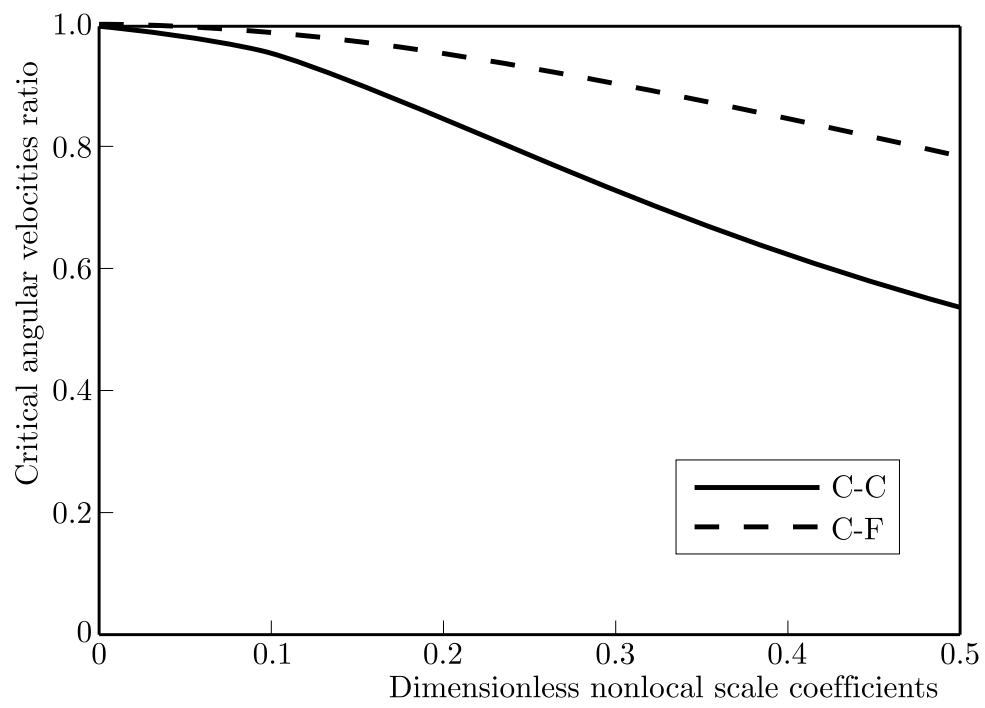

Fig. 2. Critical angular velocities ratio with dimensionless nonlocal scale coefficients

\section{Conclusions}

In this work, the static resonance phenomenon is investigated for rotating nanobars under clamped-free and clamped-clamped boundary conditions. Here, the classical linear uniaxial model is extended by adopting Eringen's nonlocal elasticity theory, and the equation of motion is formulated in the Lagrangian coordinates. If the critical angular velocities obtained from the nonlocal elasticity calculations are very small, as compared to those from the local elasticity calculations, this linear uniaxial model can be reliably used, as indicated by Hodges and Bless (1994) in detail. Hence, it should be noted that the linear uniaxial model used here will give more reliable results with an increase in the scale coefficient for nanobars with sufficiently small lengths under the clamped-clamped boundary conditions. The present analysis based on the nonlocal elasticity theory shows that the static resonance can be a primary critical case for the rotating nanobars having very small lengths, in contrast to the classical elasticity theory.

\section{References}

1. Aranda-Ruiz J., Loya J., Fernandez-SAEz J., 2012, Bending vibrations of rotating nonuniform nanocantilevers using the Eringen nonlocal elasticity theory, Composite Structures, 94, 2990-3001

2. Bhuta P.G., Jones J.P., 1963, On axial vibrations of a whirling bar, The Journal of the Acoustical Society of America, 35, 217-221

3. BRunelle E.J., 1971, Stress redistribution and instability of rotating beams and disks, America Institute Aeronautics Astronautics, 9, 758-759

4. Danesh V., Asghari M., 2014, Analysis of micro-rotating disk based on the strain gradient elasticity, Acta Mechanica, 225, 1955-1965

5. Eringen A.C., 2002, Nonlocal Continuum Field Theories, Springer, New York

6. Hodges D.H., Bless R.R., 1994, Axial instability of rotating rods revisited, International Journal of Non-linear Mechanics, 29, 879-887

7. NARENDAR S., 2011, Mathematical modelling of rotating single-walled carbon nanotubes used in nanoscale rotational actuators, Defence Science Journal, 61, 317-324 
8. NAREndar S., 2012, Differential quadrature based nonlocal flapwise bending vibration analysis of rotating nanotube with consideration of transverse shear deformation and rotary inertia, Applied Mathematics and Computation, 219, 1232-1243

9. Narendar S., Gopalakrishnan S., 2011, Nonlocal wave propagation in rotating nanotube, Results in Physics, 1, 17-25

10. Narendar S., Roy Mahapatra D., Gopalakrishnan S., 2011, Prediction of nonlocal scaling parameter for armchair and zigzag single-walled carbon nanotubes based on molecular structural mechanics, nonlocal elasticity and wave propagation, International Journal of Engineering Sciences, 49, 509-522

11. Shum W.S., Entwistle R.D., 2006, Longitudinal vibration frequencies of steadily whirling rods, The Journal of the Acoustical Society of America, 119, 909-916

Manuscript received December 1, 2017; accepted for print January 10, 2018 